\title{
Common Mathematical Model of Fatigue Characteristics
}

\author{
Z. Maléř, S. Slavík, T. Marczi, M. Růžička
}

This paper presents a new common mathematical model which is able to describe fatigue characteristics in the whole necessary range by one equation only:

$\log N=A(R)+B(R) \cdot \log S_{\mathrm{a}}$

where $A(R)=A R^{2}+B R+C$ and $B(R)=D R^{2}+A R+F$.

This model was verified by five sets of fatigue data taken from the literature and by our own three additional original fatigue sets. The fatigue data usually described the region of $N=10^{4}$ to $3 \times 10^{6}$ and stress ratio of $R=-2$ to 0.5 . In all these cases the proposed model described fatigue results with small scatter. Studying this model, following knowledge was obtained:

- the parameter "stress ratio $R$ " was a good physical characteristic

- the proposed model provided a good description of the eight collections of fatigue test results by one equation only

- the scatter of the results through the whole scope is only a little greater than that round the individual $S / N$ curve

- using this model while testing may reduce the number of test samples and shorten the test time

- as the proposed model represents a common form of the $S / N$ curve, it may be used for processing uniform objective fatigue life results, which may enable mutual comparison of fatigue characteristics.

Keywords: fatigue characteristics, mathematical model, stress ratio $R$.

\section{Introduction}

It is a great advantage if the designer has reliable fatigue characteristics of the main carrying parts while designing a new aircraft. The first complete characteristics of wings were make in internationally known laboratories. Nowadays, major producers create the characteristics of their own specific, commonly used parts. This paper offers a new mathematical model that enables fatigue tests to be considered during testing, and in this way fatigue characteristics can be established very reliably and economically. For illustration, three examples of the use of this model are shown.

\section{The present state of fatigue characteristics expression}

$S_{\mathrm{a}}$ amplitude and $S_{\mathrm{m}}$ mean stress of the load cycle are decisive and at the same time, also geometrically transparent factors influencing the life of structure elements, the influence of these quantities on the life, expressed by the number of cycles until failure $A$, is often presented graphically for the system of $S / N$ curves [1] (see Fig. 1) or in the form of the Haigh diagram [2] (see Fig. 4).

Where computers were introduced, the graphical representation of fatigue characteristics was no longer satisfactory, and mathematical ways of expression were searched. An expression used to describe the fatigue characteristics of wing and horizontal tail surfaces [1,3] was one of the first models

$$
\log N=a\left(S_{\mathrm{m}}\right)+b\left(S_{\mathrm{m}}\right) \log \left(S_{\mathrm{a}}-0.835\right)
$$

where $a\left(S_{\mathrm{m}}\right)$ and $b\left(S_{\mathrm{m}}\right)$ represent the positions (intercept on $\log N$ axis) and slopes of $S / N$ curves left branches.

NOTE: This paper will not deal with the right side of $S / N$ curves, i.e., fatigue limits.

Parameters $a\left(S_{\mathrm{m}}\right)$ and $b\left(S_{\mathrm{m}}\right)$ are functionally dependent on the mean stress value (see Fig. 1) and they are described in four segments by means of polynomials of the first and third degree.

This way of expression is accurate, and it enables a computer to be used. The width of the segments and the mathematical function are chosen to replace the results of fatigue tests as accurately as possible. However, selection of the segment width and suitable polynomials leads to the loss of the general character. The system of polynomials accurately describes the geometrical form of the results but this description has no the physical sense. The system does not describe the physical relations among $S_{\mathrm{a}}, S_{\mathrm{m}}$ and $N$.

After supplying the existing results with another group of results, or when processing another set of test results it is necessary to choose new width segments and a new system of polynomials to achieve an accurate presentation of the test results. Section widths and polynomials are chosen by way of trial.

For these reasons this model is not generally valid, nor can it be used in fatigue test design or during fatigue tests to control them. It is necessary to find a new mathematical model.

The requirements for the new mathematical model can be expressed as follows:

1. The model should have the basic form of the common model of $S / N$ curve

$$
\log N=a+b \log \left(S_{\mathrm{a}}\right)
$$

2. Functions $a$ and $b$ should always be the same for any tested structure or element within the whole study range, and only its parameters may vary.

3. Changes of equation parameters must depend upon test results only. If the test results are homogeneous and there is no deflection from the physical limits, the equation parameters cannot change considerably when increasing the number of test samples.

4. Functions $a$ and $b$ should be valid within a high-cycle area, i.e., for $N>10^{4}$. 

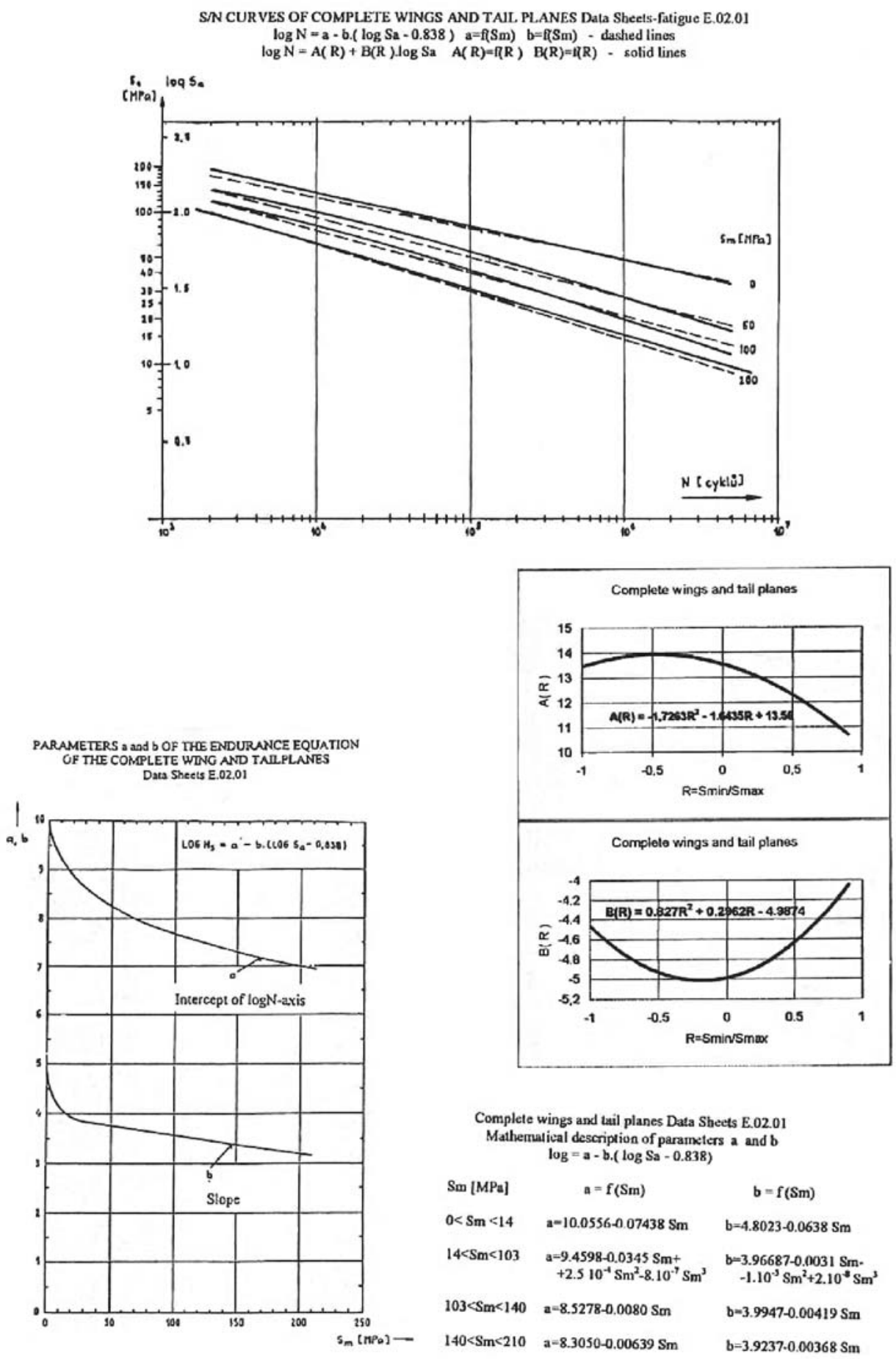

Fig. 1: $S / N$ curves of complete wings and tail plans

\section{Design of the new mathematical model}

When designing the model, we start [4] from the following knowledge. It was found out, see e.g. [5], that when describing the influenceof the mean (or better to say, at description of load cycle position influence) on crack growth, stress ratio $R$ is a more suitable parameter than the mean stress $S_{\mathrm{m}}$. For this reason parameter $R=S_{\min } / S_{\max }$ was used instead of $S_{\mathrm{m}}$. The relation among $S_{\mathrm{a}}, R, N$ is described in the shape

$$
Y=\log N=A(R)+B(R) \cdot \log S_{\mathrm{a}} .
$$

This function describes the system/family of $S / N$ curve branches that correspond to different values of $R=$ const. in the area of high-cycle fatigue, see Fig. 2. The position and the slope parameters of $\mathrm{S} / \mathrm{N}$ curve branches, in co-ordinate system $\log S_{\mathrm{a}}-\log N$, depends on the value of $R$. Dependencies $A(R)=f(R)$ and $B(R)=f(R)$ are expressed by means of the following functions:

$$
\begin{aligned}
& A(R)=A R^{2}+B R+C, \\
& B(R)=D R^{2}+E R+F .
\end{aligned}
$$




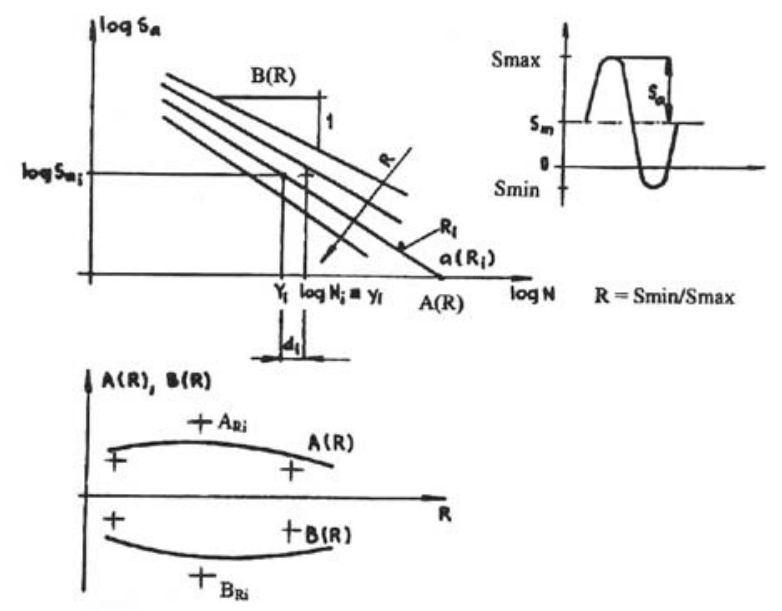

THE SCHEME AND THE EQUATION OF THE NEW MATHEMATICAL MODEL DESCRIBING THE ELEMENTARY FATIGUE CHARACTERISTICS

$\log N=A(R)+B(R) \cdot \log S a \quad A(R)=A \cdot R^{2}+B \cdot R+C$ $B(R)=D \cdot R^{2}+E \cdot R+F$

Constants A, B, ..., E are derived on the base of the test results by means of the mean square deviation method.

Fig. 2: The scheme and the equation of the new mathematical model describing the elementary fatigue characteristics

The relations for calculations parameters $A, B, C, \ldots, F$ are determined through the method of smallest squares of deviations $d_{i}$ between results $N_{i}$ and model function $Y_{i}$ :

$$
\begin{aligned}
& q=\sum_{i=1}^{n} d_{i}^{2}=\sum\left(\log N_{i}-Y_{i}\right)^{2} \rightarrow \text { minimum } \\
& \frac{\delta q}{\delta A}=0, \quad \frac{\delta q}{\delta B}=0, \quad \frac{\delta q}{\delta C}=0, \quad \ldots, \quad \frac{\delta q}{\delta F}=0
\end{aligned}
$$

As a result we obtained a system of six normal equations. These enable six parameters $A, B, C, \ldots, F$ of the model function $S_{\mathrm{a}}-R-A$ to be established.

The input values for calculating the model function parameters are $n$ triples of values $\left(S_{\mathrm{a}_{i}}, R_{i}, N_{i}\right)$, where $N_{i}$ is the number of cycles to failure achieved at the test.

NOTE: To determine parameters $A, B, \ldots, F$ it is necessary to create a computer program. Then calculating parameters $A, B$, $\ldots, F$ is easy, and so is not difficult to process fatigue the test results during testing, and thus to control these tests while they are still running. This involves altering the test plan on the basis of the obtained results, and thus reducing the number of test samples and shortening the time of testing.

At the beginning of testing it is necessary to carry out fatigue tests at a minimum of three stress ratios of $R=$ const., while at each $R$ it is necessary to perform tests a minimum of three $S_{\mathrm{a}}$ levels. The range of $R\left(R_{\min }\right.$ to $\left.R_{\max }\right)$ is established according to usual needs. The $S_{\mathrm{a}}$ levels are assessed so that the $N$ will be between $N=10^{4}$ to $10^{6}$.

On this basis, the $S_{\mathrm{a}}-R-N$ diagram can be supplemented by other tests only in regions with information shortage.

To describe the scatter of the test results round the model function (3) the following expression is used

$$
s_{F}=\left(\frac{1}{n-6} \sum\left(\log N_{i}-Y_{i}\right)^{2}\right)^{\frac{1}{2}}
$$

where $n$ is the number of test results described by $n$-triples $\left(S_{\mathrm{a}}, R, N\right), Y_{i}$ is the theoretical number of load cycles given by the model function (3). The $Y_{i}$ value corresponds to probability of failure of $p=50 \%$.

In the test on Al-alloy elements, in which the $S / N$ curve has two straight line sections of different slopes, each part of the curve must be solved separately.

\section{Examples of judgement of validity and accuracy of the $S_{\mathrm{a}}-R-N$ model}

Suggested $S_{\mathrm{a}}-R-N$ model (3) was judged on five sets of fatigue results taken from the literature, and was used for processing our own threesets of test results.

Validity and accuracy were judged by:

- comparing the origin and $S_{\mathrm{a}}-R-N$ diagrams,

- comparing $S / N$ curve parameters $\left(A R_{i}, B R_{i}\right)$ and model functions $A(R), B(R)$,

- value of standard deviation $s_{F}(6)$.

This paper will be describe the following three results.

\subsection{Example 1}

Fig. 1 shows $S / N$ curves of complete wings and tail planes. The test results there were taken as points read from the original curves. The dashed lines are the original $S / N$ curves according to the Data Sheets - Fatigue E.02.01 [1], and the solid lines are calculated by the new $S_{\mathrm{a}}-R-N$ model, see equation (3). As we can see, the differences are negligible. The equation is:

$$
\log N=A(R)+B(R) \cdot \log S_{\mathrm{a}}
$$

where

$$
\begin{aligned}
& A(R)=-1.726325 R^{2}-1.643550 R+13.560228 \\
& B(R)=+0.827044 R^{2}+0.296186 R-4.987437 .
\end{aligned}
$$

The differences between the original and the model curves are expressed by the value of the standard deviation $s_{F}=0.086$.

\subsection{Example 2}

Fig. 4 compares the original constant life curves (thin curves) of the MUSTANG wings and the curves established by the new $S_{\mathrm{a}}-R-N$ model (3) (thick curves) [2]. The model curves were calculated in the range of $N=10^{4}$ to $10^{7}$ cycles. The test results were taken as read from the original $N=$ const. curves for $R=-1 ;-0.8 ;-0.6 ; \ldots ; 0.6$.

$$
\log N=A(R)+B(R) \cdot \log S_{\mathrm{a}}
$$

where

$$
\begin{aligned}
& A(R)=0.542293 R^{2}+1.125954 R+11.914455 \\
& B(R)=-0.853812 R^{2}-1.948959 R-5.884825 .
\end{aligned}
$$

The $s_{F}$ value represents the differences/scatter between the new model and the original curves.

The value of the standard deviation is $s_{F}=0.107$.

\subsection{Example 3}

Fig. 3 there are shows our original test results for the test specimen that represents a steel attachment critical point. The specimens are made of Czech Poldi L-ROL.7 steel heat treated to $R_{m_{\min }}=1080 \mathrm{MPa}$ which is similar to U.S. 4130 alloy 

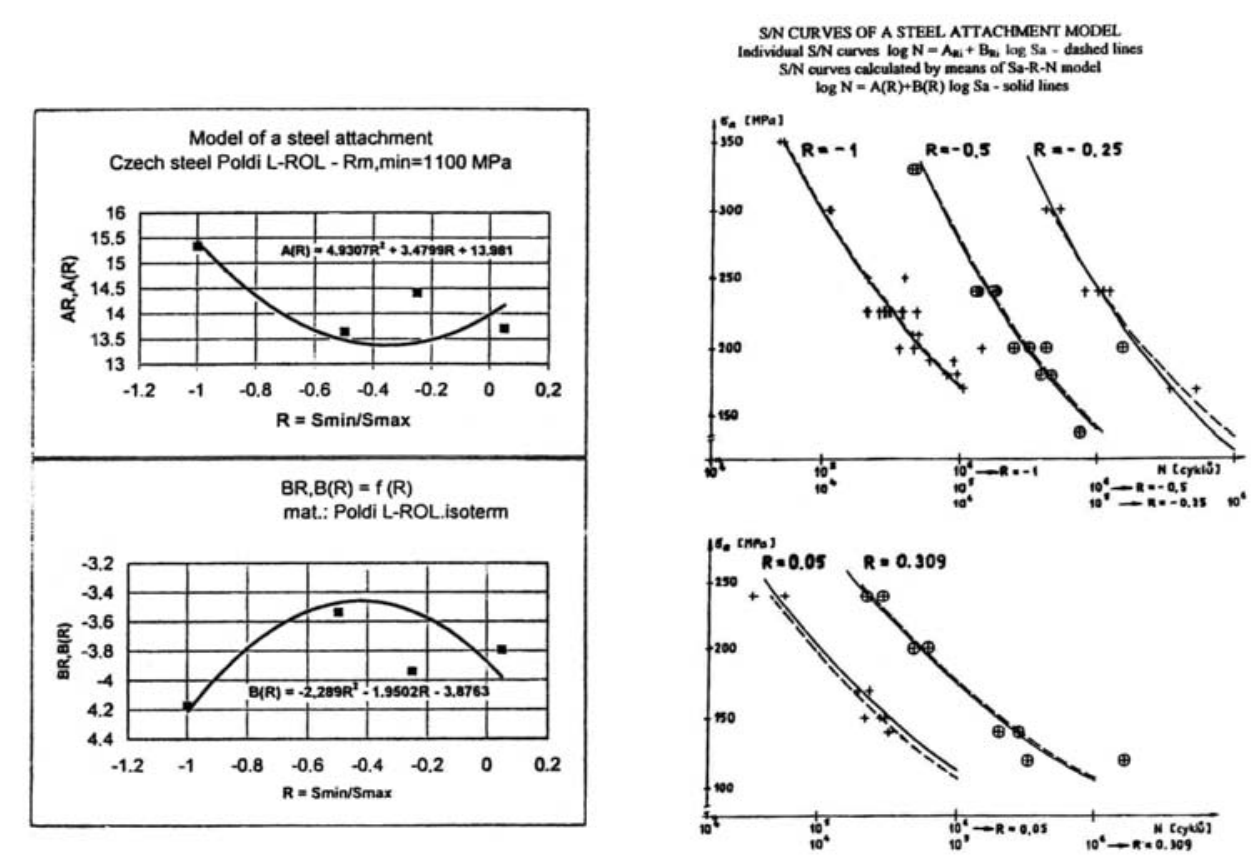

Fig. 3: The original test results for the test specimen that represents a steel attachment critical point

steel. The specimens were axially loaded at $R=-1 ;-0.5 ; 0.23$;

0.42 . The equation describing the $S_{\mathrm{a}}-R-N$ model is:

$$
\log N=A(R)+B(R) \cdot \log S_{\mathrm{a}}
$$

where

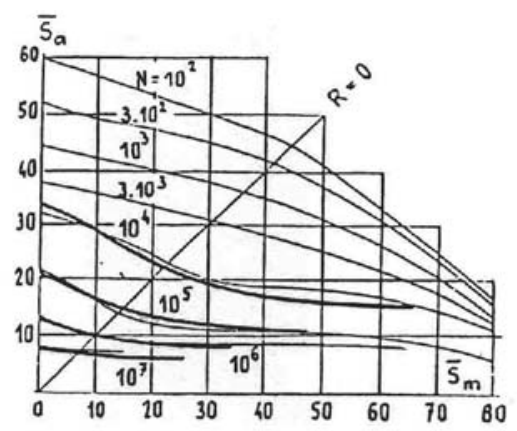

HAIGH'S DIAGRAM OF THE MUSTANG P-SID WING - thin curves are original; bold ones calculated by Sa-R-N model

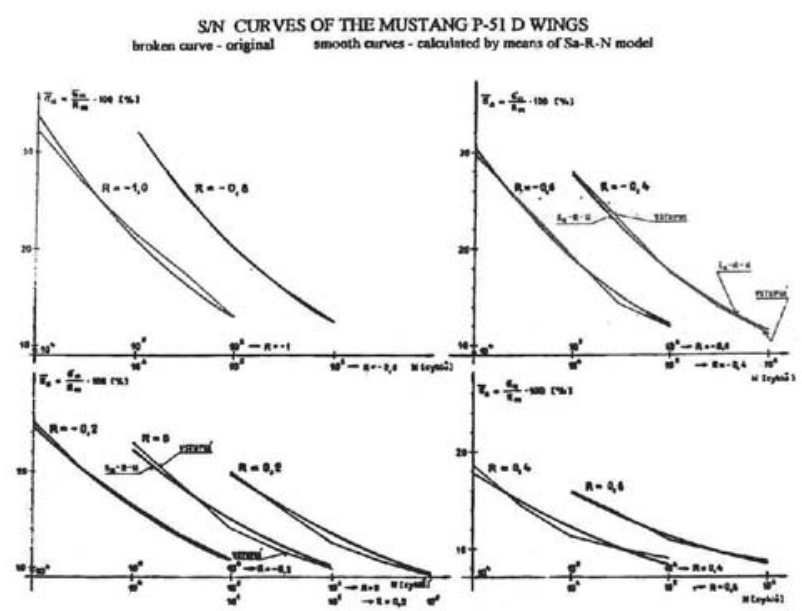

Fig. 4: The comparison of the original constant life curves (thin curves)of the MUSTANG wings and the curves established by the new $S_{\mathrm{a}}-R-N$ model

$$
\begin{aligned}
& A(R)=4.930716 R^{2}+3.479890 R+13.980960 \\
& B(R)=-2.289047 R^{2}-1.950201 R-3.876255 .
\end{aligned}
$$

The standard deviation of the test results round the $S_{\mathrm{a}}-R-N$ model is $s_{F}=0.167$.
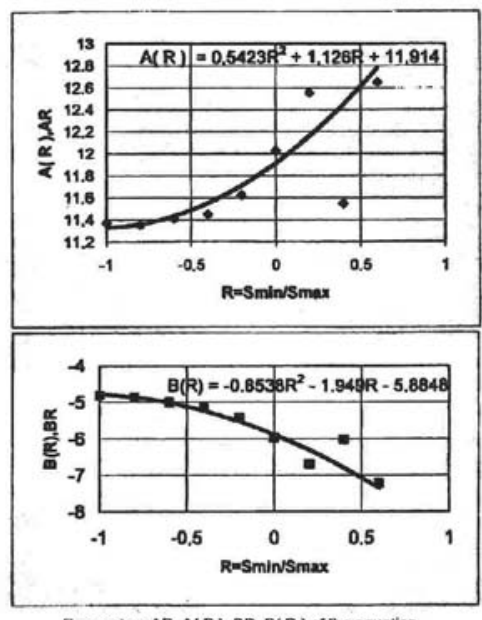
MUSTANG WINGS
MUS arameters $A R, A(R), B R, B(R)$ of the equation 


\section{Partial conclusion}

This paper presents three of the eight test groups on which the new $S_{\mathrm{a}}-R-N$ model has been tested. In all five other checked fatigue sets the new model describes the test results with similar precision and in the whole test range by one equation of (3) only.

\section{Conclusion}

The following conclusions can be drawn from a comparison of the original characteristics and the characteristics obtained when using the mathematical model (3):

1. The designed model was able to describe, with sufficient accuracy and reliability, the results for five different structures and elements within the range of $N=10^{4}$ through $10^{6}$ to $10^{7}$, and within the range of $R=-1$ through 0.6 , pre-processed in some way (graphical or mathematical).

2. When processing the original results of the fatigue tests, the $S / N$ curves determined through model function (3) were nearly identical with partial $S / N$ curves calculated for different values of $R=$ const. The scatter of test results calculated from the whole set was not much higher than that around the individual $S / N$ curves.

3. Parameter $R$ (together with $S_{\mathrm{a}}$ ) proved to be a suitable physical parameter for describing the load cycle.

Further knowledge follows from this:

4. The $S_{\mathrm{a}}-R-N$ model can be considered as a suitable, generally valid mathematical description of fatigue characteristics within the high-cycle fatigue area $\left(N>10^{4}\right)$.

5. For this reason, model (3) enables economical design of fatigue tests, because during tests not just one $S / N$ curve but the wide region s described. The number of test samples is not much greater.

6. As the designed model is only a generalized form of a commonly used $S / N$ curve, it provides a basis for uniform, comparable, objective and accurate processing of fatigue test results.

7. Instead of $S_{\mathrm{a}}, S_{\max }$ can also be used.

\section{Acknowledgment}

This paper is presented thanks to the Airspace Research Center at the Institute of Aerospace Engineering at the Technical University in Brno and at the Czech Technical University in Prague, Czech Republic.

\section{References}

[1] Engineering Science Data Unit, (Data Sheets - Fatigue E.02.01.): Endurance for Complete Wing and Tail Structures. Royal Aeronautical Society, 1958.

[2] Johnstone W. W., Payne A. O.: Aircraft Structural Fatigue Research in Australia Fatigue in Aircraft Structures. Proceedings of the International Conference (Ed.: A. M. Freudenthal). Columbia University, 1956, Fig. on page 436.

[3] Hangartner R.: "Correlation of Fatigue Data for Aluminium Aircraft Wing and Tail Structures". National Aeronautical Establishment. Ottawa: 1974, Aeronautical Report LR-582.

[4] Maléř Z.: Basis for Calculation Estimate of Safe Life of the primary structure of a Small Airplane . Doctor thesis, Military Academy, Brno (Czech Republic), 1990.

[5] Hudson C. M.: Effect of Stress Ratio on Fatigue Crack Growth.Report NASA Res.Center, Bethlehem, 1967.

Ing. Zdeněk Maléř, CSc.

Private adviser in fatigue life

U Trojáku 4650

76005 Zlín, Czech Republic

Doc. Ing. Svatomír Slavík, CSc.

phone: +420224357227

e-mail: svatomir.slavik@fs.cvut.cz

Ing. Tomáš Marczi

phone: +420224357433

e-mail: tomas.marczi@fs.cvut.cz

Department of Auto motive and Airspace Eng.

Czech Technical University in Prague

Faculty of Mechanical Engineering

Karlovo náměstí 13

12135 Praha 2, Czech Republic

Doc. Ing. Milan Růžička, CSc.

phone: +420224352512

e-mail: milan.ruzicka@fs.cvut.cz

Department of Mechanics

Czech Technical University in Prague

Faculty of Mechanical Engineering

Technická 4

16607 Praha 6, Czech Republic 